\title{
THE PROGENITOR OF SUPERNOVA 2011dh HAS VANISHED
}

\author{
Schuyler D. Van Dyk ${ }^{1}$, WeiKang Zheng ${ }^{2}$, Kelsey I. Clubb ${ }^{2}$, Alexei V. Filippenko ${ }^{2}$, S. Bradley Cenko ${ }^{2,3}$, \\ Nathan Smith ${ }^{4}$, Ori D. Fox ${ }^{2}$, Patrick L. Kelly ${ }^{2}$, IsaAC Shivvers ${ }^{2}$, and Mohan Ganeshalingam ${ }^{5}$ \\ ${ }^{1}$ Spitzer Science Center/Caltech, Mailcode 220-6, Pasadena, CA 91125, USA; vandyk@ipac.caltech.edu \\ ${ }^{2}$ Department of Astronomy, University of California, Berkeley, CA 94720-3411, USA \\ ${ }^{3}$ Astrophysics Science Division, NASA Goddard Space Flight Center, Mail Code 661, Greenbelt, MD 20771, USA \\ ${ }^{4}$ Steward Observatory, University of Arizona, Tucson, AZ 85720, USA \\ ${ }^{5}$ Lawrence Berkeley National Laboratory, Berkeley, CA 94720, USA \\ Received 2013 May 14; accepted 2013 July 3; published 2013 July 19
}

\begin{abstract}
We conducted Hubble Space Telescope (HST) Snapshot observations of the Type IIb supernova (SN) 2011dh in M51 at an age of $\sim 641$ days with the Wide Field Camera 3 . We find that the yellow supergiant star, clearly detected in pre-SN HST images, has disappeared, implying that this star was almost certainly the progenitor of the SN. Interpretation of the early time SN data which led to the inference of a compact nature for the progenitor, and to the expected survival of this yellow supergiant, is now clearly incorrect. We also present ground-based $U B V R I$ light curves obtained with the Katzman Automatic Imaging Telescope at Lick Observatory up to SN age $\sim 70$ days. From the light-curve shape including the very late time HST data, and from recent interacting binary models for SN 2011dh, we estimate that a putative surviving companion star to the now deceased yellow supergiant could be detectable by late 2013, especially in the ultraviolet. No obvious light echoes are detectable yet in the SN environment.
\end{abstract}

Key words: galaxies: individual (NGC 5194) - stars: evolution - supernovae: general - supernovae: individual (SN 2011dh)

\section{INTRODUCTION}

Supernova (SN) 2011dh (also known as PTF11eon) in Messier 51 (M51; more precisely, M51a or NGC 5194) is a nearby example of the intermediate class of core-collapse supernovae ( $\mathrm{SNe}$ ), the Type IIb, existing between the hydrogen-rich SNe II and the hydrogen-stripped SNe Ib (see Filippenko 1997 for a review of SN classification). The progenitors of SNe II, especially the Type II-Plateau SNe (SNe II-P; the most common core-collapse events), have been shown to be red supergiants (RSGs) through direct progenitor identifications (e.g., Smartt et al. 2004; Fraser et al. 2011; Van Dyk et al. 2012a, 2012b). The RSG progenitors of SNe II-P appear to be consistent with expectations of single-star evolution models (e.g., Falk \& Arnett 1977; Wheeler \& Swartz 1993; Dessart \& Hillier 2011). The progenitors of both $\mathrm{SNe} \mathrm{Ib}$ and $\mathrm{SNe} \mathrm{IIb}$, on the other hand, require the outer stellar envelope to be substantially stripped away prior to explosion. One possible channel has long been thought to be massive interacting binary systems (e.g., Podsiadlowski et al. 1993; Woosley et al. 1994; Maund et al. 2004; Claeys et al. 2011). SNe IIb thus provide us with vital information regarding the evolutionary transition from a single massive RSG, with relatively low late-stage mass loss, to a star for which the mass loss before explosion must have been far more vigorous, potentially as a result of mass exchange with a companion. SNe IIb constitute about $10 \%-11 \%$ of all core collapse SNe (Smith et al. 2011; Li et al. 2011), and their implied mass range appears to be consistent with binary evolution. Chevalier \& Soderberg (2010) have further separated the already rare $\mathrm{SNe}$ IIb into those whose progenitors are compact (radius $\left.R \approx 10^{11} \mathrm{~cm}\right)$ and those that are extended $\left(R \approx 10^{13} \mathrm{~cm}\right)$, based on emission from the shock-heated envelope and the radio and $\mathrm{X}$-ray properties of the SNe. Hence, all nearby, well-studied cases of SNe IIb are particularly valuable.

SN 2011dh was discovered independently by several amateur astronomers (as summarized by Griga et al. 2011) and by the
Palomar Transient Factory collaboration (Arcavi et al. 2011) within $\sim 1$ day of explosion, between May 31 and June 1 . Early photometry and spectra of the SN were presented by Arcavi et al. (2011), who argued that the data, when compared to analytical models of SN shock breakout, were inconsistent with an extended progenitor. Based on early-time radio and X-ray data, Soderberg et al. (2012) also reasoned that the progenitor must have been compact. Further radio observations were compiled by Krauss et al. (2012), Bietenholz et al. (2012), and Horesh et al. (2012). Additional X-ray data were collected and analyzed by Horesh et al. (2012), Campana \& Immler (2012), and Sasaki \& Ducci (2012). Krauss et al. and Bietenholz et al. maintained that the progenitor was compact. Horesh et al. concluded that the uncertainties in the modeling of the existing $\mathrm{X}$-ray and radio data were larger than previously estimated, and therefore the inferred progenitor radius is consistent both with being compact (as found for the SN IIb 2008ax; Pastorello et al. 2008; Taubenberger et al. 2011; Chornock et al. 2011) and extended (best exemplified by the SN IIb 1993J in M81; e.g., Richmond et al. 1996; Matheson et al. 2000).

Shortly after discovery of SN 2011dh, Li \& Filippenko (2011) identified a possible progenitor star in archival Hubble Space Telescope (HST) images of M51 obtained with the Advanced Camera for Surveys (ACS). This identification was confirmed by both Maund et al. (2011) and Van Dyk et al. (2011) by comparing ground-based adaptive optics images of the $\mathrm{SN}$ with the archival HST ACS and Wide Field Planetary Camera 2 (WFPC2) images. The candidate progenitor had the bolometric luminosity $\left(L_{\text {bol }} \approx 10^{5} L_{\odot}\right)$ and effective temperature $\left(T_{\text {eff }} \approx 6000 \mathrm{~K}\right)$ of a yellow supergiant (YSG). Van Dyk et al. (2011), following the conclusions of Arcavi et al. (2011) and Soderberg et al. (2012), assumed that the progenitor star was compact and speculated that the YSG was the companion in an interacting binary system to the hotter, undetected progenitor. Van Dyk et al. concluded that the YSG's initial mass was $M_{\text {initial }}=17-19 M_{\odot}$, suggested by the star's locus in the Hertzsprung-Russell diagram (HRD) 

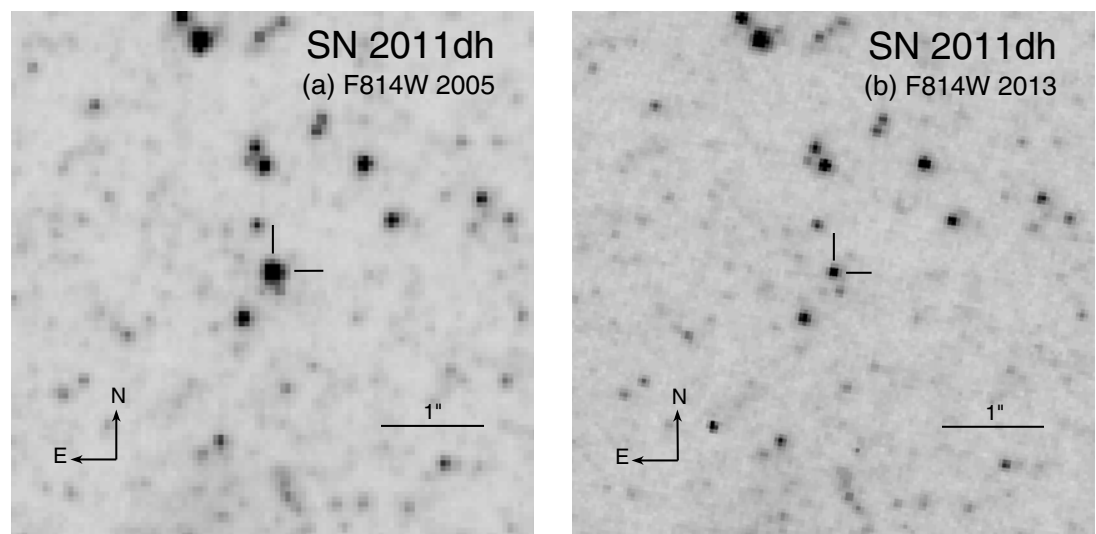

Figure 1. (a) A portion of the archival HST ACS F814W image of M51 from 2005; the progenitor of SN 2011dh is indicated by tick marks. (b) A portion of the HST WFC3 F814W image from 2013, to the same scale and orientation, and approximately the same contrast level; the SN is also indicated by tick marks. North is up and east is to the left.

compared with the tracks of luminosity and effective temperature for single massive-star theoretical evolutionary models. In contrast, Maund et al. (2011) concluded that the YSG was, in fact, the likely progenitor star, with $M_{\text {initial }}=13 \pm 3 M_{\odot}$, comparing its locus in the HRD only to the endpoint luminosities of the evolutionary tracks. Murphy et al. (2011) analyzed the stellar populations around the progenitor and found that if the YSG had indeed vanished, then the progenitor likely had $M_{\text {initial }} \approx 13 M_{\odot}$, in agreement with Maund et al.

Bersten et al. (2012) subsequently performed hydrodynamical modeling of the existing light curves of SN 2011dh. In particular, they showed that a more extended progenitor (with radius $R \approx 200 R_{\odot}$ ) was necessary to account for the post-shockbreakout thermal cooling and luminosity decline within the first $\sim 4$ days after explosion, indicated by the early $g^{\prime}$-band data from Arcavi et al. (2011). This implies that the progenitor star at explosion was a supergiant with a low-mass $\mathrm{H}$ envelope $\left(\sim 0.1 M_{\odot}\right)$. Bersten et al. concluded from their models that the progenitor's initial mass was consistent with $12-15 M_{\odot}$. The He core mass in the models was $\sim 4 M_{\odot}$, which, as Bersten et al. pointed out, is more consistent with the lower core mass expected from an interacting binary model than with a singlestar progenitor model. However, the presence of a putative companion was entirely hidden by the light from the supergiant; both Maund et al. (2011) and Van Dyk et al. (2011) showed that the observed spectral energy distribution of the supergiant could account for all of the flux in each HST band, including the ultraviolet (UV) F336W band.

Benvenuto et al. (2013) continued exploring the binary scenario. For assumed initial masses of $16 M_{\odot}$ for the YSG primary (donor) star and $10 M_{\odot}$ for the secondary (a mass, and therefore luminosity, low enough to allow the secondary to remain undetected even in the pre-SN UV image), and an initial orbital period of 125 days, these authors found that, through 3 episodes of mass transfer, the primary explodes with properties similar to those observed for the identified YSG and the secondary remains near a hot zero-age main sequence temperature and luminosity. The mass, temperature, and luminosity of the secondary were all found to increase as the mass-transfer efficiency in the model interacting binary system was increased.

Ultimately, the key to determining the nature of the progenitor is to observe the $\mathrm{SN}$ at sufficiently late times to reveal that the progenitor no longer is there. This sort of analysis has been conducted in recent years to a good effect by Maund \&
Smartt (2009 for SNe 1993J and 2003gd), Gal-Yam \& Leonard (2009 for SN 2005gl), Maund et al. (2013 in particular, for SNe 2004A, 2005cs, and 2006my), and Van Dyk (2013 for SN 2008bk). In this Letter we show that the YSG at the position of SN 2011dh has, in fact, disappeared (see also Van Dyk et al. 2013 where we initially announced this discovery). We also present multi-band photometry for the $\mathrm{SN}$ from day 5 to day 70 using the $0.76 \mathrm{~m}$ Katzman Automatic Imaging Telescope (KAIT; Filippenko et al. 2001) at Lick Observatory. UT dates are used throughout (i.e., UTC, which is an approximation for UT1).

\section{OBSERVATIONS AND ANALYSIS}

We observed SN 2011dh on 2013 March 2.44 (at an age of 641 days) with HST using the Wide Field Camera 3 (WFC3) UVIS channel and filters F555W and F814W. These observations are part of our Cycle 20 Snapshot program GO13029 (PI: A. V. Filippenko). We display in Figure 1(b) the SN as seen in F814W. For comparison, we show in panel (a) the HST ACS F814W image of the progenitor from 2005, with the same scale and orientation, and approximately the same contrast; the figure is similar to the one presented by Van Dyk et al. (2011; their Figure 1).

We extracted photometry from the WFC3 images using Dolphot v2.0 (Dolphin 2000). We present the HST flightsystem magnitudes for the $\mathrm{SN}$ in Table 1. We have also remeasured photometry for the progenitor from the $2005 \mathrm{HST}$ ACS and WFPC2 images presented by Van Dyk et al. (2011; also see Maund et al. 2011) using this version of Dolphot (we had employed version 1.1 in Van Dyk et al. 2011). We also used the pixel-based charge-transfer-efficiency-corrected ACS images available in the $H S T$ archive. The star is now measured to be somewhat brighter in all bands, although the differences at $\mathrm{F} 336 \mathrm{~W}$ and $\mathrm{F} 814 \mathrm{~W}$ are within the uncertainties (which are rather large at F336W and very small at F814W). This new photometry is presented in Table 1 . From the table one can see that the SN in 2013 March is 1.39 and 1.30 mag fainter in F555W and F814W, respectively, than was the YSG.

We have also observed the SN with KAIT in $U B V R I$ between 2011 June 3.25 (day 5) and August 8.18 (day 70). We first subtracted away the light from the host galaxy using template images in BVRI obtained at the Lick Observatory Nickel $1 \mathrm{~m}$ telescope on 2013 March 13 and in $U$ on 2013 May 8, when the $\mathrm{SN}$ was no longer detectable in these 
Table 1

HST Photometry at the Position of SN 2011 dh ${ }^{\mathrm{a}}$

\begin{tabular}{|c|c|c|c|c|c|c|c|c|}
\hline Epoch & $\begin{array}{c}\text { F336W } \\
\text { (mag) }\end{array}$ & $\begin{array}{c}\text { F435W } \\
\text { (mag) }\end{array}$ & $\begin{array}{c}B \\
(\mathrm{mag})\end{array}$ & $\begin{array}{c}\text { F555W } \\
\text { (mag) }\end{array}$ & $\begin{array}{c}V \\
(\mathrm{mag})\end{array}$ & $\begin{array}{c}\text { F658N } \\
\text { (mag) }\end{array}$ & $\begin{array}{c}\text { F814W } \\
\text { (mag) }\end{array}$ & $\begin{array}{c}I \\
(\mathrm{mag})\end{array}$ \\
\hline 2005 & $23.380(283)$ & $22.368(005)$ & 22.380 & $21.813(006)$ & 21.759 & $21.166(017)$ & $21.211(005)$ & 21.203 \\
\hline
\end{tabular}

Note. ${ }^{\text {a }}$ Uncertainties $(1 \sigma)$ are given in parentheses as millimagnitudes.

Table 2

KAIT Photometry of SN 2011 dh

\begin{tabular}{lccccc}
\hline \hline JD-2,400,000 & $\begin{array}{c}U \\
(\mathrm{mag})\end{array}$ & $\begin{array}{c}B \\
(\mathrm{mag})\end{array}$ & $\begin{array}{c}V \\
(\mathrm{mag})\end{array}$ & $\begin{array}{c}R \\
(\mathrm{mag})\end{array}$ & $\begin{array}{c}I \\
(\mathrm{mag})\end{array}$ \\
\hline 55715.75 & $14.82(08)$ & $15.33(08)$ & $14.82(05)$ & $14.56(05)$ & $14.37(03)$ \\
55719.77 & $14.39(07)$ & $14.49(08)$ & $13.95(06)$ & $13.62(06)$ & $13.60(05)$ \\
55720.73 & $14.17(07)$ & $14.36(09)$ & $13.67(05)$ & $13.43(05)$ & $13.40(04)$ \\
55721.70 & $\ldots$ & $14.15(09)$ & $13.52(08)$ & $13.28(06)$ & $13.20(03)$ \\
55722.72 & $\ldots$ & $14.00(09)$ & $13.30(05)$ & $13.05(06)$ & $13.02(04)$ \\
55723.73 & $\ldots$ & $13.75(07)$ & $13.17(05)$ & $12.92(04)$ & $12.88(04)$ \\
55725.85 & $\ldots$ & $13.54(06)$ & $\ldots$ & $\ldots$ & $\ldots$ \\
55726.72 & $\ldots$ & $13.59(07)$ & $12.86(04)$ & $12.68(04)$ & $12.57(04)$ \\
55728.72 & $13.50(13)$ & $13.54(08)$ & $12.78(05)$ & $12.56(06)$ & $12.42(03)$ \\
55729.73 & $13.42(07)$ & $13.61(09)$ & $12.66(03)$ & $12.51(06)$ & $12.36(02)$ \\
55731.73 & $13.42(06)$ & $13.28(02)$ & $12.57(02)$ & $12.34(02)$ & $12.23(02)$ \\
55738.72 & $\ldots$ & $13.80(04)$ & $12.84(04)$ & $12.42(03)$ & $12.17(03)$ \\
55748.73 & $15.84(09)$ & $14.93(07)$ & $13.64(03)$ & $13.00(03)$ & $12.62(03)$ \\
55751.70 & $15.99(09)$ & $14.99(06)$ & $13.89(09)$ & $13.25(08)$ & $12.74(05)$ \\
55754.72 & $\ldots$ & $\ldots$ & $13.97(06)$ & $13.36(08)$ & $12.86(05)$ \\
55757.70 & $\ldots$ & $15.26(05)$ & $14.05(06)$ & $13.34(03)$ & $12.89(02)$ \\
55760.72 & $16.14(08)$ & $15.31(06)$ & $14.08(03)$ & $13.47(05)$ & $12.98(02)$ \\
55763.70 & $\ldots$ & $15.26(05)$ & $14.16(04)$ & $13.55(05)$ & $13.05(04)$ \\
55766.72 & $\ldots$ & $15.32(04)$ & $14.24(05)$ & $13.61(05)$ & $13.14(04)$ \\
55769.72 & $\ldots$ & $15.42(05)$ & $14.31(04)$ & $13.67(04)$ & $13.18(04)$ \\
55772.71 & $\ldots$ & $15.42(05)$ & $14.34(04)$ & $13.79(06)$ & $13.24(06)$ \\
55775.71 & $\ldots$ & $\ldots$ & $14.38(03)$ & $13.79(04)$ & $\ldots$ \\
55781.68 & $16.23(10)$ & $15.47(05)$ & $14.46(05)$ & $13.95(06)$ & $13.38(04)$ \\
& & & & &
\end{tabular}

Note. ${ }^{\text {a }}$ Uncertainties $(1 \sigma)$ are given in parentheses as hundredths of a magnitude.

ground-based images. The templates were astrometrically registered, rescaled to match the pixel size of the KAIT images, and sky-background matched before subtraction. Point-spread function (PSF) photometry was applied using the DAOPHOT (Stetson 1987) package from IDL Astronomy User's Library. ${ }^{6}$ The instrumental magnitudes and colors of the SN were transformed at BVRI to the standard Johnson-Cousins system using the photometric sequence around the host galaxy presented by Ergon et al. (2013a; their Table A.5), specifically their stars P092, P09-3, P09-4, P09-A, and E13-1. At $U$ we used only the two brightest of these stars, P09-2 and P09-4. Uncertainties in the calibration of our photometry are $0.03 \mathrm{mag}$ in $U, 0.02 \mathrm{mag}$ in $B$, and $0.01 \mathrm{mag}$ in $V R I$, and these have been added in quadrature with the measurement uncertainties.

We present the KAIT photometry in Table 2 and display the resulting light curves in Figure 2. We also show for comparison the expected light-curve decline rate $\left(0.98 \mathrm{mag}(100 \text { day })^{-1}\right)$ powered by the radioactive decay of ${ }^{56} \mathrm{Co}$.

Tsvetkov et al. (2012) present UBVRI photometry for the SN over the first 300 days. Marion et al. (2013) and Ergon et al. (2013a) also provide UV-to-infrared photometry and spectroscopy of SN 2011dh over its first 34 and 100 days,

\footnotetext{
6 http://idlastro.gsfc.nasa.gov/contents.html
}

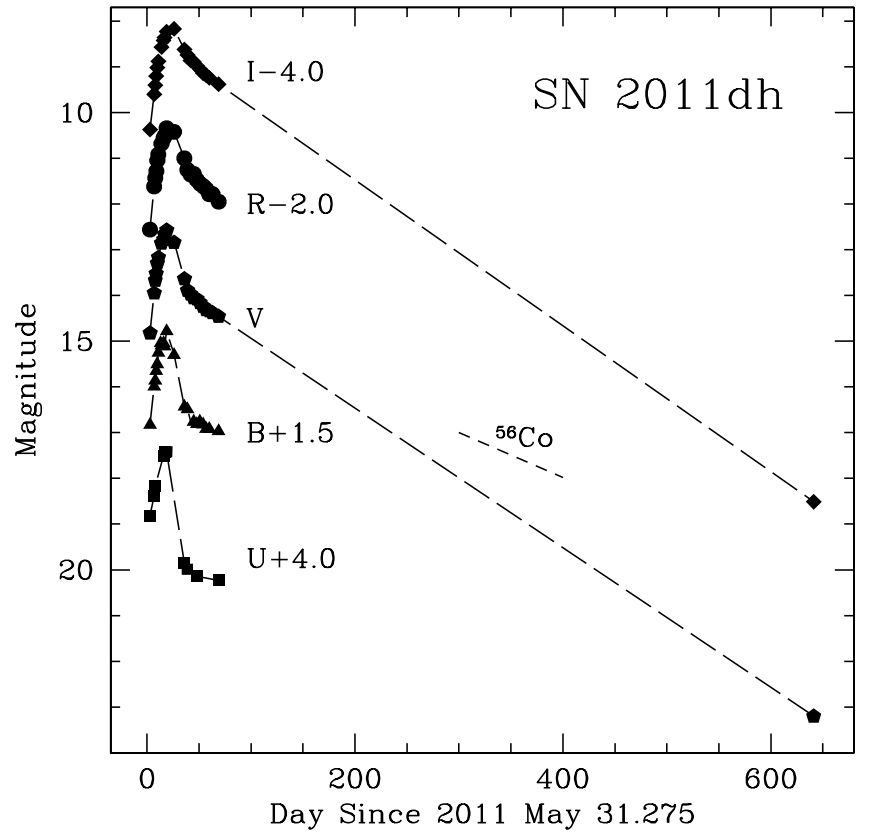

Figure 2. Light curves of SN 2011dh, including data points from KAIT and $H S T$. Also shown is the expected decline rate from the radioactive decay of ${ }^{56} \mathrm{Co}$ (short-dashed line). We interpolate between the last data points with KAIT to the HST data points with the long-dashed line. The HST WFC3 F555W and F814W are assumed to be $\sim V$ and $\sim I$ bands.

respectively. Our photometry compares quite well with that of Tsvetkov et al.: differences are $\ll 0.1 \mathrm{mag}$ for $B V R I$, and our photometry in $U$ agrees very well through maximum light, but is $\lesssim 0.4$ mag brighter post-maximum. A similar favorable comparison also exists with the data from Ergon et al. (2013a), although our $B$ photometry is $\sim 0.2$ mag brighter after day $\sim 38$, and our $U$ photometry is systematically $\sim 0.2 \mathrm{mag}$ brighter. The remaining discrepancies in $U$ between our photometry and that of Tsvetkov et al. and Ergon et al. could result from differences in application of a color term, which we did not apply. More significant differences are generally found with the Marion et al. data set: our photometry is $0.8-1.0 \mathrm{mag}$, 0.2-0.4 mag, 0.15-0.2 mag, and 0.2-0.3 mag brighter in $U$, $B, V$, and $I$, respectively, and $\lesssim 0.4$ mag brighter than the Swift $U$ photometry (a discrepancy which could result from differences in calibration); the best agreement is in $R$, with differences of $\ll 0.1 \mathrm{mag}$. Overall, we cannot provide an explanation for the differences between our photometry and that presented by Marion et al.

\section{DISCUSSION AND CONCLUSIONS}

It is evident from our recent HST imaging that the YSG has vanished, meaning that this is almost certainly the star that actually exploded, in agreement with the theoretical analyses by Bersten et al. (2012) and Benvenuto et al. (2013), and 
with the conclusions of Maund et al. (2011) and Horesh et al. (2012). A similar conclusion was reached by Ergon et al. (2013a, 2013b) from their ground-based imaging. Possible loci of the progenitor and its companion in the HRD are shown by Benvenuto et al. (2013). The model YSG progenitor of SN 2011dh from Bersten et al. (2012) has a radius $R \approx 200 R_{\odot}$, which is less extended than the progenitor of the best-studied SN IIb 1993J: assuming the inferred absolute $V$ magnitude and $\mathrm{K} 0$ spectral type (effective temperature $\sim 4200 \mathrm{~K}$, with corresponding bolometric correction from Levesque et al. 2005) for the SN 1993J supergiant progenitor from Van Dyk et al. (2002), that star's radius was $\sim 580 R_{\odot}$. However, the SN 2011dh progenitor is far more extended than, say, the radius inferred by Chevalier \& Soderberg $\left(2010, \sim 10^{11} \mathrm{~cm}\right)$ for the SN IIb 2008ax compact progenitor (see, however, Horesh et al. 2012 on the uncertainties in distinguishing between compact and extended progenitors based on radio data alone). We speculate that the origin of compact versus extended progenitors of SNe IIb, if this distinction actually exists, may arise in the initial conditions of the binary system (assuming the binary hypothesis applies for these $\mathrm{SNe}$ ) and the extent of mass exchange between the components. A more complete discussion of SN IIb progenitors is beyond the scope of this Letter.

We interpolate in Figure 2 between the end of our photometric coverage of the SN with KAIT to the HST WFC3 data points. The behavior of the light curves shows that the SN light declined over $\sim 641$ days more rapidly $\left(\sim 0.015 \mathrm{mag} \mathrm{day}^{-1}\right.$ in $V$ and $\left.I\right)$ than expected from ${ }^{56} \mathrm{Co}$ decay, possibly as a result of increasing transparency of the SN ejecta to the $\gamma$-rays emitted from the decay (e.g., Arnett \& Fu 1989) or from dust formation.

We detect nebular emission lines from the $\mathrm{SN}$ in recent optical spectra; Shivvers et al. (2013) present our analysis of them and discuss implications for the nature of the progenitor star. These spectra show that light from the $\mathrm{SN}$ is still dominant at the time of the WFC3 observations. The $\mathrm{SN}$ is therefore currently too bright for any binary companion of the progenitor to be detected.

However, we can estimate the earliest date at which the companion, if it exists, could become visible. If we adopt the mass-transfer efficiency of 0.25 from Benvenuto et al. (2013; their Table 3), the secondary has $T_{\text {eff }} \approx 30,000 \mathrm{~K}$, $L_{\text {bol }}=10^{4.13} L_{\odot}$, and surface gravity $\log g \approx 4.3$ (essentially that of a late $\mathrm{O}$ - or early B-type supergiant). Assuming a bolometric correction for a supergiant at this temperature from Flower (1996), an M51 distance of 8.4 Mpc from Vinkó et al. (2012), and an extinction $A_{V}<0.25$ mag (adopting the Galactic foreground contribution from Schlafly \& Finkbeiner 2011 and the upper limit on the host-extinction contribution from Arcavi et al. 2011, with $R_{V}=3.1$ ), we find that the secondary's brightness would be $V \lesssim 27.2 \mathrm{mag}$. (We note that Bersten et al. 2012 also estimate that the progenitor's companion should have a visual magnitude of 26-27.) Given the current decline rate of the $\mathrm{SN}$, we estimate that a surviving companion might become visible at day $\sim 900$ — that is, as early as 2013 mid-November. Furthermore, given the expected high effective temperature of the surviving companion, a search for this star is optimized in the UV, since the star's light should dominate at these wavelengths. If the $\mathrm{SN}$ in the $U$ band has been declining at the same rate as in $V$ and $I$, then by day $\sim 900$ the SN should have become quite faint, at $U \approx 29 \mathrm{mag}$. (One caveat here is that the postmaximum $U$-band light curves shown by Tsvetkov et al. 2012 and especially Ergon et al. 2013a appear to flatten out up to day 100.) Also, from Benvenuto et al. (2013), if the mass-transfer efficiency (essentially a free parameter) is higher, detection would be possible sooner, since the star should be hotter and more luminous; however, if it is lower, we may have to wait longer to detect the star.

Finally, we note that no obvious, large-scale light echo has yet appeared in the 2013 HST images. Assuming the VEGAMAG zero point for WFC3/UVIS ${ }^{7}$ and a plate scale of $0^{\prime \prime} .04$ pixel $^{-1}$, we place a $3 \sigma$ upper limit on the surface brightness of an extended echo at F555W of $\gtrsim 21.6 \mathrm{mag} \operatorname{arcsec}^{-2}$. (We also cannot rule out that a more compact echo, within the image PSF, is contributing to the observed SN flux.) However, the extended echoes around the SN IIb 1993J were not detected until $\sim 8$ yr after explosion (Sugerman \& Crotts 2002; Liu et al. 2003; echoes were not detectable $\sim 2$ yr post-explosion). Thus, it is possible we may still see one or more echoes emerge in future $H S T$ images of SN 2011dh, particularly in the UV and blue bands.

This work is based in part on observations made with the NASA/ESA Hubble Space Telescope, obtained from the Data Archive at the Space Telescope Science Institute (STScI), which is operated by the Association of Universities for Research in Astronomy (AURA), Inc., under NASA contract NAS 0526555. KAIT and its ongoing research were made possible by donations from Sun Microsystems, Inc., the Hewlett-Packard Company, AutoScope Corporation, Lick Observatory, the NSF, the University of California, the Sylvia \& Jim Katzman Foundation, and the TABASGO Foundation. Support for this research was provided by NASA through grants AR-12623 and GO13029 from STScI. A.V.F. and his group at UC Berkeley also wish to acknowledge generous support from Gary and Cynthia Bengier, the Richard and Rhoda Goldman Fund, the Christopher R. Redlich Fund, the TABASGO Foundation, and NSF grant AST-1211916.

Facilities: HST (ACS, WFC3), KAIT

\section{REFERENCES}

Arcavi, I., Gal-Yam, A., Yaron, O., et al. 2011, ApJL, 742, L18

Arnett, W. D., \& Fu, A. 1989, ApJ, 340, 396

Benvenuto, O. G., Bersten, M. C., \& Nomoto, K. 2013, ApJ, 762, 74

Bersten, M. C., Benvenuto, O. G., Nomoto, K., et al. 2012, ApJ, 757, 31

Bietenholz, M. F., Brunthaler, A., Soderberg, A. M., et al. 2012, ApJ, 751, 125 Campana, S., \& Immler, S. 2012, MNRAS, 427, L70

Chevalier, R. A., \& Soderberg, A. M. 2010, ApJL, 711, L40

Chornock, R., Filippenko, A. V., Li, W., et al. 2011, ApJ, 739, 41

Claeys, J. S. W., de Mink, S. E., Pols, O. R., Eldridge, J. J., \& Baes, M 2011, A\&A, 528, A131

Dessart, L., \& Hillier, D. J. 2011, MNRAS, 410, 1739

Dolphin, A. E. 2000, PASP, 112, 1383

Ergon, M., Sollerman, J., Fraser, M., et al. 2013a, A\&A, submitted (arXiv:1305.1851)

Ergon, M., Sollerman, J., Pursimo, T., et al. 2013b, ATel, 4912, 1

Falk, S. W., \& Arnett, W. D. 1977, ApJS, 33, 515

Filippenko, A. V. 1997, ARA\&A, 35, 309

Filippenko, A. V., Li, W. D., Treffers, R. R., \& Modjaz, M. 2001, in ASP Conf. Ser. 246, Small-Telescope Astronomy on Global Scales, ed. W. P. Chen, C. Lemme, \& B. Paczyński (San Francisco, CA: ASP), 121

Flower, P. J. 1996, ApJ, 469, 355

Fraser, M., Ergon, M., Eldridge, J. J., et al. 2011, MNRAS, 417, 1417

Gal-Yam, A., \& Leonard, D. C. 2009, Natur, 458, 865

Griga, T., Marulla, A., Grenier, A., et al. 2011, CBET, 2736

Horesh, A., Stockdale, C., Fox, D. B., et al. 2012, arXiv:1209.1102

Krauss, M. I., Soderberg, A. M., Chomiuk, L., et al. 2012, ApJL, 750, L40

Levesque, E. M., Massey, P., Olsen, K. A. G., et al. 2005, ApJ, 628, 973

Li, W., \& Filippenko, A. V. 2011, CBET, 2736

\footnotetext{
http://www.stsci.edu/hst/wfc3/phot_zp_lbn
} 
Li, W., Leaman, J., Chornock, R., et al. 2011, MNRAS, 412, 1441

Liu, J.-F., Bregman, J. N., \& Seitzer, P. 2003, ApJ, 582, 919

Marion, G. H., Vinko, J., Kirshner, R. P., et al. 2013, ApJ, submitted (arXiv:1303.5482)

Matheson, T., Filippenko, A. V., Barth, A. J., et al. 2000, AJ, 120, 1487

Maund, J., Reilly, E., \& Mattila, S. 2013, MNRAS, submitted (arXiv:1302.7152)

Maund, J. R., Fraser, M., Ergon, M., et al. 2011, ApJL, 739, L37

Maund, J. R., \& Smartt, S. J. 2009, Sci, 324, 486

Maund, J. R., Smartt, S. J., Kudritzki, R. P., Podsiadlowski, P., \& Gilmore, G. F. 2004, Natur, 427, 129

Murphy, J. W., Jennings, Z. G., Williams, B., Dalcanton, J. J., \& Dolphin, A. E. 2011, ApJL, 742, L4

Pastorello, A., Kasliwal, M. M., Crockett, R. M., et al. 2008, MNRAS, 389, 955

Podsiadlowski, P., Hsu, J. J. L., Joss, P. C., \& Ross, R. R. 1993, Natur, 364, 509

Richmond, M. W., Treffers, R. R., Filippenko, A. V., \& Paik, Y. 1996, AJ, 112,732

Sasaki, M., \& Ducci, L. 2012, A\&A, 546, A80

Schlafly, E. F., \& Finkbeiner, D. P. 2011, ApJ, 737, 103

Shivvers, I., Mazzali, P., Silverman, J. M., et al. 2013, MNRAS, submitted (arXiv:1307.2246)
Smartt, S. J., Maund, J. R., Hendry, M. A., et al. 2004, Sci, 303, 499

Smith, N., Li, W., Filippenko, A. V., \& Chornock, R. 2011, MNRAS, 412, 1522

Soderberg, A. M., Margutti, R., Zauderer, B. A., et al. 2012, ApJ, 752, 78

Stetson, P. B. 1987, PASP, 99, 191

Sugerman, B. E. K., \& Crotts, A. P. S. 2002, ApJL, 581, L97

Taubenberger, S., Navasardyan, H., Maurer, J. I., et al. 2011, MNRAS, 413,2140

Tsvetkov, D. Yu., Volkov, I. M., Sorokina, E., et al. 2012, PZ, 32, 6

Van Dyk, S. D. 2013, AJ, 146, 24

Van Dyk, S. D., Cenko, S. B., Poznanski, D., et al. 2012a, ApJ, 756, 131

Van Dyk, S. D., Davidge, T. J., Elias-Rosa, N., et al. 2012b, AJ, 143, 19

Van Dyk, S. D., Filippenko, A. V., Fox, O., et al. 2013, ATel, 4850, 1

Van Dyk, S. D., Garnavich, P. M., Filippenko, A. V., et al. 2002, PASP, 114,1322

Van Dyk, S. D., Li, W., Cenko, S. B., et al. 2011, ApJL, 741, L28

Vinkó, J., Takáts, K., Szalai, T., et al. 2012, A\&A, 540, A93

Wheeler, J. C., \& Swartz, D. A. 1993, SSRv, 66, 425

Woosley, S. E., Eastman, R. G., Weaver, T. A., \& Pinto, P. A. 1994, ApJ, 429, 300 